micropenis, and distinct anomalies of the hands and feet. Another example of Wiedemann syndrome? Clin Genet Aug 1994;46:205-208). (Respond: Professor NC Nevin, Department of Medical Genetics, Floor A, Lisburn Road, Belfast BT9 7AB, UK).

COMMENT. Wiedemann and associates (1985) described 2 males, first cousins, with similar characteristics, whose mothers and maternal grandfather had short broad thumbs and halluces. The present case, the second report of this syndrome, had normal parents. Autosomal or Xlinked dominant inheritance was thought to be most consistent with the findings.

\title{
LIFE EXPECTANCY WITH CEREBRAL PALSY
}

The life expectancy of children with idiopathic cerebral palsy born during 1966-84 to mothers resident in Mersey region has been analysed at the Department of Public Health, University of Liverpool, UK. Among 1251 subjects traced, one third had quadriplegia, one third hemiplegia, a quarter had diplegia, and the remainder dyskinesia and ataxia. A quarter had severe ambulatory disability, a fifth severe manual disability, and a third an IQ of 50 or less; $11 \%$ had died. One third had a birth weight of $2500 \mathrm{~g}$ or less, and one third a gestational age of 37 weeks or less. Those with normal birth weight had the highest proportion of severe disabilities. About $85-90 \%$ of subjects with CP survived to 20 years compared to $97 \% 20$ year survival in the general population in 1970-2. Subjects with mild functional disabilities (ambulation, manual dexterity, and IQ deficits) had 20 year survival of $99 \%$, while those severely disabled had a 50\% 20 year survival. Birth weight and gestational age were less predictive of survival than functional disability. (Hutton JL et al. Life expectancy in children with cerebral palsy. BMI 13 August 1994;309:431435). (Respond: Professor Peter OD Pharoah, Department of Public Health, University of Liverpool, Liverpool L69 3BX, UK).

COMMENT. One-half of severely disabled cerebral palsied children survived to age 20 , and the life expectancy to 20 years of mild to moderately disabled children was not much lower than that of unaffected children. As more severely affected low birth weight infants survive with advances in neonatal care, future cohorts may show a higher proportion of severely disabled with $\mathrm{CP}$ and a life expectancy approaching that of the normal birth weight CP children. The social, educational, health service, and medico-legal aspects of these findings are noted. A much shorter life expectancy is reported in US studies.

\section{BRAIN DEVELOPMENT MEASURED BY MRI}

MRI data read as normal on 88 male and female patients aged 3 months to 30 years and on 73 healthy male volunteers aged 21 to 70 years were quantified and the volumes of cortical white matter, gray matter, and CSF were computed in a study at the Department of Veterans Affairs Medical Center, and the Department of Psychiatry and Behavioral Science, Stanford University School of Medicine, CA. In the younger samples, obtained from four California clinics, intracranial volume increased by about $300 \mathrm{ml}$ from 3 months to 10 years. Head size of boys was larger than that of girls by about $70 \mathrm{~mL}$, but both sexes followed the same growth trend. Cortical gray matter volume peaked at age 4 years and decreased thereafter; cortical white matter volume increased steadily until age 20 years: cortical and ventricular CSF volumes remained constant. In the older sample, cortical gray matter volume decreased 
curvilinearly by $0.7 \mathrm{~mL} /$ year, while white matter volume remained constant through 5 decades. Cortical CSF volume increased by $0.6 \mathrm{~mL} / \mathrm{y}$ and ventricular volumes increased by $0.3 \mathrm{~mL} / \mathrm{y}$ as cortical gray matter decreased. (Pfefferbaum A et al. A quantitative magnetic resonance imaging study of changes in brain morphology from infancy to late adulthood. Arch Neurol Sept 1994;51:874-887). (Reprints: Dr Pfefferbaum, Psychiatric Service (116A3), Palo Alto Department of Veterans Affairs Medical Center, 3801 Miranda Ave, Palo Alto, CA 94304).

COMMENT. Age-related changes in gray-white matter ratio suggest that growth in white matter exceeds that of gray during the first 5 years, continues to expand until age 20 years, whereas gray matter volume declines after age 5 . Age 4 years marks the end of gray matter growth and the beginning of a consistent decline throughout the life span. A relation between head size and cortical gray matter is established early and persists into late adulthood. These quantitative studies of normal brain development, reflecting cell growth and death, myelination, and atrophy, provide important comparative data in the investigation of neurodegenerative processes.

\section{DEGENERATIVE DISORDERS}

\section{MACHADO-JOSEPH DISEASE}

A 22-year-old male of Portuguese Azorean descent, presenting at age 16 years with postural instability and falls and developing severe generalized dystonia by age 20 years, is reported from the Center for Research in Neurodegenerative Diseases, University of Toronto, Ontario, Canada. His parents were first cousins and each had a parent clinically affected by Machado-Joseph disease (MJD). Examination demonstrated in addition to dystonia, slurred speech, horizontal nystagmus, limitation of upward-gaze, unsustained ankle clonus, and flexor plantar reflexes. MRI revealed slight atrophy of the cerebellar vermis. Linkage studies confirmed the recent mapping of the MJD gene to chromosome 14q, and genotyping of the members of this pedigree indicated that this patient was homozygous for the MJD gene. Gene dosage is an important determinant of age at onset and clinical phenotype in MJD. (Lang AE et al. Homozygous inheritance of the MachadoJoseph disease gene. Ann Neurol Sept 1994;36:443-447). (Respond: Dr Lang, Morton and Gloria Shulman Movement Disorder Centre, The Toronto Hospital, Western Division, MP11-306, 399 Bathurst Street, Toronto, Ontario, Canada M5T 2S8).

COMIMENT. Three major phenotypes of MJD are described: Type I, Joseph type, with early age of onset and prominent extrapyramidal signs dystonia, athetosis, rigidity, as well as pyramidal signs; Type III, Machado type, with later onset, cerebellar signs and peripheral neuropathy; and Type II, intermediate type, both with respect to age of onset and clinical features. Juvenile onset of MJD is very uncommon, occurring in only 5 of 143 Portuguese patients cited by these authors. 